\title{
Fast and Longest Rollercoasters
}

\section{Paweł Gawrychowski $^{1} \cdot$ Florin Manea $^{2}$ (D) Radosław Serafin ${ }^{1}$}

Received: 9 August 2019 / Accepted: 27 November 2021 / Published online: 17 February 2022

(c) The Author(s) 2022

\section{Abstract}

For $k \geq 3$, a $k$-rollercoaster is a sequence of numbers whose every maximal contiguous subsequence, that is increasing or decreasing, has length at least $k$; 3-rollercoasters are called simply rollercoasters. Given a sequence of distinct real numbers, we are interested in computing its maximum-length (not necessarily contiguous) subsequence that is a $k$-rollercoaster. Biedl et al. (in: ICALP, volume 107 of LIPIcs. Schloss Dagstuhl - Leibniz-Zentrum fuer Informatik, pp 18:1-18:15, 2018) have shown that each sequence of $n$ distinct real numbers contains a rollercoaster of length at least $\lceil n / 2\rceil$ for $n>7$, and that a longest rollercoaster contained in such a sequence can be computed in $O(n \log n)$-time (or faster, in $O(n \log \log n$ ) time, when the input sequence is a permutation of $\{1, \ldots, n\})$. They have also shown that every sequence of $n \geq(k-1)^{2}+1$ distinct real numbers contains a $k$-rollercoaster of length at least $\frac{n}{2(k-1)}-\frac{3 k}{2}$, and gave an $O(n k \log n)$-time (respectively, $O(n k \log \log n)$-time) algorithm computing a longest $k$-rollercoaster in a sequence of length $n$ (respectively, a permutation of $\{1, \ldots, n\})$. In this paper, we give an $O\left(n k^{2}\right)$-time algorithm computing the length of a longest $k$-rollercoaster contained in a sequence of $n$ distinct real numbers; hence, for constant $k$, our algorithm computes the length of a longest $k$-rollercoaster in optimal linear time. The algorithm can be easily adapted to output the respective $k$-rollercoaster. In particular, this improves the results of Biedl et al. (2018), by showing that a longest rollercoaster can be computed in optimal linear time. We also present an algorithm computing the length of a longest $k$-rollercoaster

A preliminary version of this paper was presented at the 36th International Symposium on Theoretical Aspects of Computer Science, STACS 2019.

$凶$ Florin Manea

florin.manea@cs.uni-goettingen.de

Paweł Gawrychowski

gawry@cs.uni.wroc.pl

Radosław Serafin

radserafin@gmail.com

1 Institute of Computer Science, University of Wrocław, Wrocław, Poland

2 Institute of Computer Science and Campus Institute Data Science, University of Göttingen, Goldschmidtstraße 7, 24118 Göttingen, Germany 
in $O\left(n \log ^{2} n\right)$-time, that is, subquadratic even for large values of $k \leq n$. Again, the rollercoaster can be easily retrieved. Finally, we show an $\Omega(n \log k)$ lower bound for the number of comparisons in any comparison-based algorithm computing the length of a longest $k$-rollercoaster.

Keywords Sequences - Alternating runs · Patterns in permutations · Rollercoasters · Efficient algorithms

\section{Introduction}

The mathematical study of patterns occurring in sequences of numbers is a rather old and well developed topic in combinatorics and algorithms on sequences. Within this topic, of a particularly high interest is the study of long increasing and decreasing (not necessarily contiguous) subsequences occurring in a sequence. For example, already in 1749, Euler defined the Eulerian polynomials, which are the generating function for the number of descents in permutations. Almost 200 years later, Erdős and Szekeres [11] proved the existence of an increasing or a decreasing subsequence of length at least $a+1$ in a sequence of at least $n=a^{2}+1$ distinct reals. More precisely, they have shown the following theorem.

Theorem 1 (Erdős and Szekeres [11]) Every sequence of $a b+1$ distinct real numbers contains an increasing subsequence of length at least $a+1$ or a decreasing subsequence of length at least $b+1$.

The theorem of Erdős-Szekeres is strongly related to, and in fact also follows from, the well-known decomposition of Dilworth (see [19]) regarding chains and antichains in a finite partially ordered set. Dilworth's result can be restated in the context of the combinatorics of patterns in sequences of numbers as follows.

Theorem 2 (Dilworth [10]) Any finite sequence $S$ of distinct real numbers can be partitioned into $k$ ascending sequences, where $k$ is the maximum length of a descending sequence in $S$.

Recent surveys on the combinatorics of patterns occurring in sequences are $[15,16]$.

The study of patterns in sequences of numbers also has a well developed algorithmic side (see, e.g., $[4,9,12,14]$ ). For instance, finding a longest increasing subsequence (not necessarily contiguous) contained in the input sequence is a basic problem in theoretical computer science, studied already from the 1960s [3,17,18], with applications in areas such as bioinfomatics and physics (see [20] and the references therein). In particular, Fredman [12] presented an algorithm (which he attributed to Knuth, now considered folklore) computing the length of a longest increasing subsequence (LIS) in an array of $n$ numbers in $O(n \log n)$ time, and proved that this is optimal for comparison-based algorithms. If required, the algorithm can be extended to retrieve such a subsequence. If the input sequence can be sorted in linear time (in particular, when the input sequence is a permutation of $\{1, \ldots, n\})$ and we do not require the algorithm to be comparison-based, the solution given by Fredman can be implemented in $O(n \log \log n)$ time, see [9] and the references therein. Fredman's algorithm is often 

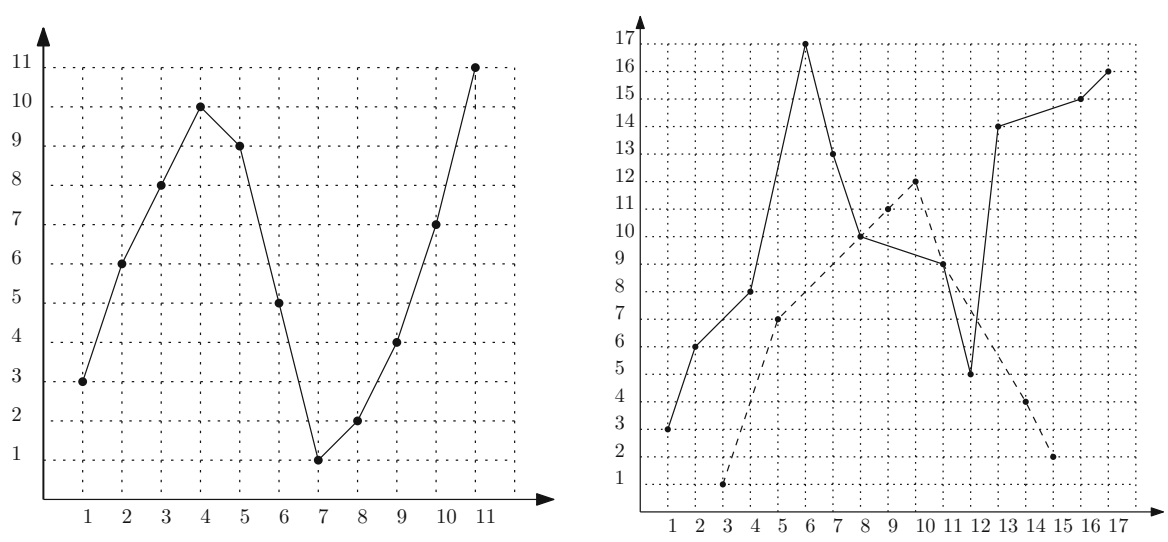

Fig. 1 Left: a 4-rollercoaster $(3,6,8,10,9,5,1,2,4,7,11)$ with runs $(3,6,8,10),(10,9,5,1)$, $(1,2,4,7,11)$. Right: two 4-rollercoasters, represented with a solid and, respectively, a dashed line, in $(3,6,1,8,7,17,13,10,11,12,9,5,14,4,2,15,16)$

called PATIENCE SORTING, and has some connections to constructing the so-called Young Tableaux $[3,17]$.

We consider a notion that is strongly related to longest increasing subsequences (and longest decreasing subsequences). A run in a sequence of numbers is a maximal contiguous subsequence that is either increasing or decreasing. A $k$-rollercoaster, where $k \geq 3$, is a sequence of numbers whose every run has length at least $k$ (i.e., the run contains at least $k$ elements); 3-rollercoasters are called, for short, rollercoasters. For example, the sequence $A=(3,6,8,10,9,5,1,2,4,7,11)$ has the runs $(3,6,8,10)$, $(10,9,5,1),(1,2,4,7,11)$; as all these runs have at least 4 elements, so the length of each of them is at least 4 , and, consequently, $A$ is a 4-rollercoaster. It is important to note that two consecutive runs in a sequence share an element: the ending element of one is the starting element of the next one; this element counts when computing the length of both runs which contain it.

Given a sequence $S[1: n]=(S[1], S[2], \ldots, S[n])$ of $n$ distinct numbers, the $k$-rollercoaster problem is to find a maximum-size set of indices $\left\{i_{1}, i_{2}, \ldots, i_{m}\right\}$ such that $1 \leq i_{1}<i_{2}<\cdots<i_{m} \leq n$ and $\left(S\left[i_{1}\right], S\left[i_{2}\right], \ldots, S\left[i_{m}\right]\right)$ is a $k$-rollercoaster. In other words, this problem asks for a longest $k$-rollercoaster contained in the input sequence $S$.

There is a simple, but useful, geometrical interpretation of $k$-rollercoasters. The input sequence $S[1: n]$ can be depicted as a set $P$ of points in the plane by translating, for $i$ from 1 to $n$, the number $S[i]$ to a point $p_{i}=(i, S[i])$. In this setting, a $k$-rollercoaster in $S$ translates to a polygonal path in the plane, whose vertices are points of $P$, and such that every maximal sub-path, with positive- or negativesloped edges, has at least $k$ points. The rollercoaster $(3,6,8,10,9,5,1,2,4,7,11)$ is depicted in the left half of Fig. 1. Two 4-rollercoasters occurring in the sequence $(3,6,1,8,7,17,13,10,11,12,9,5,14,4,2,15,16)$ are depicted in the right half of the same figure. 
While rollercoasters seem interesting on their own as a combinatorial structure, the original motivation for their study was a connection to computational geometry and graph drawing, namely to point-set embeddings of caterpillars (see [5-7] and the references therein). More precisely, constructing a long rollercoaster in a sequence of numbers was used as an intermediate step towards obtaining a method of drawing a $n$-vertex top-view caterpillar, with L-shaped edges, on a set of $\frac{25}{3} n$ general orthogonal position points in the plane. This is currently the best known bound on the number of points required to draw such a graph.

In [5], and its extended version [6], the following results regarding $k$-rollercoasters were shown. First, from a combinatorial point of view, for $k=3$, it was shown that the length of a longest rollercoaster contained in a sequence of $n \geq 7$ distinct numbers is at least $\left\lceil\frac{n}{2}\right\rceil$; this is also a tight bound. As far as $k$-rollercoasters are concerned, it was shown that for $k \geq 4$ every sequence of $n \geq(k-1)^{2}+1$ distinct numbers contains a $k$-rollercoaster of length at least $\frac{n}{2(k-1)}-\frac{3 k}{2}$. From an algorithmic point of view, both previously mentioned results were constructive, leading to an $O(n)$-time (respectively $O(n \log k))$ algorithm computing a long (but not necessarily a longest) rollercoaster (respectively, $k$-rollercoaster) contained in a sequence of $n$ distinct numbers. A longest rollercoaster contained in such a sequence was computed by an extension of Fredman's algorithm in $O(n \log n)$-time, and if the input sequence is a permutation of $\{1, \ldots, n\}$ (or, more generally, sortable in linear time) in $O(n \log \log n)$ time. By further generalising this approach, an $O(n k \log n)$-time (respectively, $O(n k \log \log n)$-time) algorithm computing a longest $k$-rollercoaster in a sequence of $n$ distinct numbers (respectively, a permutation of $\{1, \ldots, n\}$ ) can be obtained. Note that, by the theorem of Erdös and Szekeres, a sequence of $n$ distinct numbers always contains a $\lfloor\sqrt{n}\rfloor$-rollercoaster.

Our Contributions We consider the problem of computing a longest $k$-rollercoaster in an input sequence $S[1: n]$ and provide three results.

Firstly, we design a comparison-based algorithm computing the length of a longest $k$-rollercoaster in a sequence of $n$ distinct numbers in $O\left(n k^{2}\right)$ time. Thus, we obtain an optimal linear-time algorithm for constant values of $k$, in particular for $k=3$. This significantly improves the results of [5,6] and shows that, even though longest rollercoasters are related to longest increasing subsequences, the rich combinatorial structure of the former makes them provably easier to find. The starting point of our algorithm is the following natural dynamic programming formulation. For each $i \leq k$, and for each position $j$ of our input sequence $S$, we compute a longest (not necessarily contiguous) subsequence of $S$ ending with $S[j]$ and with every run of length at least $k$, except for the last run, which has only $i$ elements if $i<k$ and at least $k$ elements if $i=k$. Now the difficulty is to find the predecessor $S\left[j^{\prime}\right]$ of $S[j]$ in such a subsequence in time proportional to $k$, in particular avoiding any kind of binary search. We greedily decompose the input sequence into blocks with a certain property related to Dilworth's theorem and prove, by a careful case analysis, that $j^{\prime}$ must belong to the previous few such blocks. This, together with the special structure of the blocks and appropriate data structures, allows us to find $j^{\prime}$ in $O(k)$ amortised time.

Secondly, we focus on the case of large $k$. Given that both the previous and the new algorithm have at least linear dependency on $k$, we are interested in seeing whether we can design an algorithm whose running time is independent on $k$. We design a 
subquadratic algorithm that computes a longest $k$-rollercoaster in a sequence of $n$ distinct numbers, for any $k$, in $O\left(n \log ^{2} n\right)$ time. To obtain this result, we exploit the fact that if an increasing (respectively, decreasing) run in a longest $k$-rollercoaster extends from $S[i]$ to $S[j]$, then that run should be LIS (respectively, longest decreasing sequence, LDS for short) in $S[i: j]$. If one arranges the length of LIS (respectively, LDS) in $S[i: j]$ in an $n \times n$ matrix then the matrix has the anti-Monge property. It is known that all row maxima of an anti-Monge matrix can be found in $O(n)$ time [2], that is, in sublinear time w.r.t. the size of the matrix (given an oracle access to the elements of the matrix). Such properties have been successfully exploited to speed up certain dynamic programming algorithms [21,22]. We also follow this route, and construct a longest $k$-rollercoaster using dynamic programming, essentially by gluing together LISs and LDSs of consecutive contiguous subsequences of $S$.

Thirdly, we show that any comparison-based algorithm computing a longest $k$ rollercoaster needs $\Omega(n \log k)$ comparisons. Our reasoning is similar to the one used by Fredman [12] to show that any comparison-based algorithm computing a LIS needs $\Omega(n \log n)$ comparisons. We leave as an open problem to close the gap between the lower and upper bounds shown here.

The paper is organised as follows. After a series of preliminaries, we describe the $O\left(n k^{2}\right)$-time algorithm for computing the length of a longest $k$-rollercoaster, followed by the $O\left(n \log ^{2} n\right)$-time algorithm. Then we show how the respective longest $k$-rollercoasters can be effectively constructed. We conclude with the lower bound for the number of comparisons needed to compute the length of a longest $k$-rollercoaster in a sequence of length $n$.

\section{Preliminaries}

We consider sequences of distinct real numbers and work in the comparison-based model. If $S$ is a sequence of $n$ numbers, then $|S|=n$ is the length of the sequence, and $S[i]$ denotes its $i^{\text {th }}$ element. A subsequence of $S$ is a sequence $\left(S\left[i_{1}\right], S\left[i_{2}\right], \ldots, S\left[i_{m}\right]\right)$, defined by specifying the indices $i_{1}, i_{2}, \ldots, i_{m}$ with $1 \leq i_{1}<i_{2}<\cdots<i_{m} \leq n$. For $1 \leq i \leq j \leq n, S[i: j]$ denotes the contiguous subsequence ( $S[i], S[i+$ $1], \ldots, S[j])$; in particular, $S[1: n]$ denotes the entire $S$. Note that unless explicitly stated, a subsequence is not necessarily contiguous. An increasing subsequence (respectively, decreasing subsequence) of $S$ is a subsequence $\left(S\left[i_{1}\right], S\left[i_{2}\right], \ldots, S\left[i_{m}\right]\right)$ such that $S\left[i_{j}\right]<S\left[i_{j+1}\right]$, for all $1 \leq j \leq m-1$ (respectively, $S\left[i_{j}\right]>S\left[i_{j+1}\right]$, for all $1 \leq j \leq m-1$ ). A longest increasing (respectively, decreasing) sequence, for short LIS (respectively, LDS), is an increasing (respectively, decreasing) sequence with the largest possible length. Fredman [12] gave an $O(n \log n)$-time algorithm for computing the length of LIS, denoted res in Algorithm 1. Clearly, this algorithm can be modified to compute the LDS instead of the LIS.

We recall how Algorithm 1 works. While going through the positions $i$ of $S$, from 1 to $n$, we maintain, for each $k \leq n$, the value $R[k]$ which is the smallest ending value of increasing subsequence of length $k$ contained in $S[1: i]$ ends. To maintain these values we exploit the fact that $R$ is increasing. Thus, when considering $S[i]$, we can update the array $R$ as follows. Firstly, we binary search for the maximum $k$ such that 


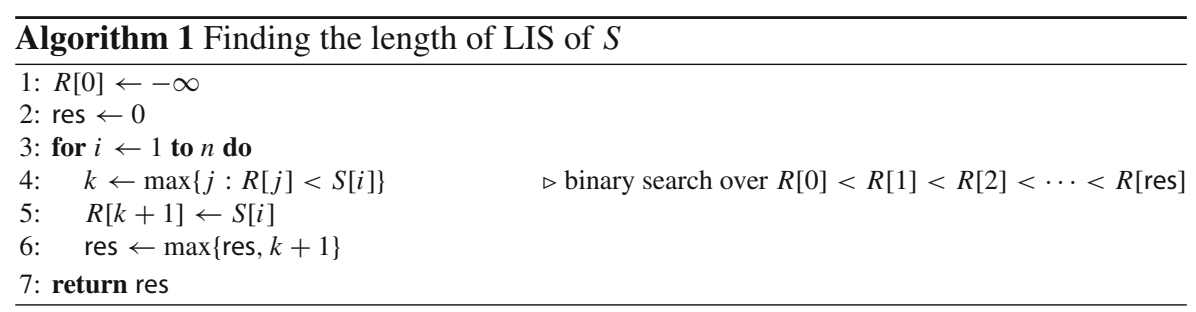

$R[k]<S[i]$. In this way, we obtain that the smallest ending value of an increasing subsequence of length $k+1$ contained in $S[1: i-1]$ is larger than $S[i]$, and, for all $k^{\prime} \leq k$, there is an increasing subsequence of length $k^{\prime}$, contained in $S[1: i-1]$, which ends with a value smaller than $S[i]$. Consequently, we conclude that the smallest ending value of an increasing subsequence of length $k+1$ contained in $S[1: i]$ should be $S[i]$ (so we set $R[k+1] \leftarrow S[i]$ ). Also, all the values $R\left[k^{\prime}\right]$, with $k^{\prime} \leq k$, should be left unchanged. Finally, all the values $R\left[k^{\prime}\right]$, with $k^{\prime}>k+1$, should also be left unchanged: otherwise, we would have had a an increasing subsequence of length $k+1$ contained in $S[1: i-1]$ ending with a value smaller than $S[i]$. After considering all the positions $i$ of $S$, we just return res, which is the largest index $k$ for which $R[k]$ was set during this algorithm.

A byproduct of this algorithm is a partition of $S[1: n]$ into res non-increasing subsequences that can be obtained by creating, for every $1 \leq j \leq$ res, a list of elements that has been stored in $R[j]$.

A run in a sequence of numbers is a maximal contiguous subsequence that is increasing or decreasing. A $k$-rollercoaster is a sequence of numbers such that every run has length at least $k$; 3-rollercoasters are called, for short, rollercoasters. Given a sequence $S[1: n]$ we are interested in finding its longest subsequence that is a $k$-rollercoaster. To make the exposition easier to follow, we focus first on finding the length of such a subsequence. Recovering the subsequence itself is, in all our algorithms, rather straightforward, and explained in Sect. 5.

\section{Computing a Longest $k$-Rollercoaster in $O\left(n k^{2}\right)$-Time}

In this section we show how to find a longest $k$-rollercoaster in a given array $S[1: n]$ in $O\left(n k^{2}\right)$ time. The algorithm we design has two parts, a preprocessing phase and a main phase, and requires some combinatorial observations. Our presentation of the algorithm is, accordingly, structured in three subsections. In the first subsection, we present our preprocessing phase. In the second subsection, we present two combinatorial lemmas which enable us to use the structures obtained in the preprocessing in a clever way in the main phase. In the third subsection, we finally describe the main phase of the algorithm.

\subsection{Preprocessing Phase}

As said, we begin our algorithm with a preprocessing phase. 
An alternating $k$-decomposition of $S[1: n]$ is a partition of $S[1: n]$ into contiguous subsequences (called parts) $S_{1}, S_{2}, \ldots, S_{m}$ such that the length of LIS in the odd parts ( $S_{1}, S_{3}, S_{5}$, and so on) is $k$ while the length of LDS in the even parts is $k$, possibly smaller for the very last part, and additionally by removing the last element of any odd (even) part we obtain a sequence with LIS (LDS) of length less than $k$. In other words, for $\ell \geq 1, S_{\ell}$ is either the shortest contiguous subsequence of $S$ that follows directly after $S_{1} \ldots S_{\ell-1}$ and has for $\ell$ odd (even) a LIS (respectively, LDS) of length $k$, if such a subsequence exists, or the whole remaining part of $S$ otherwise. For example, an alternating 3-decomposition of $S=(1,4,2,5,8,7,6,3,11,9,10,14,16,13,15,12)$ is $(1,4,2,5),(8,7,6),(3,11,9,10)$, and $(14,16,13,15,12)$.

Lemma 1 An alternating $k$-decomposition of $S[1: n]$ can be found in $O(n \log k)$ time.

Proof By terminating Algorithm 1 as soon as res $=k$ we can find the shortest prefix of $S$ with LIS equal to $k$ in $O(d \log k)$ time, where $d$ is the length of the prefix. Then we find the shortest prefix of the remaining suffix of $S$ with LDS equal to $k$, and repeat. Overall, this takes $O(n \log k)$ time because all parts are disjoint.

By Dilworth's Theorem [10], a part with LIS of length $k$ can be decomposed into $k$ decreasing subsequences, and such a decomposition can be obtained as a byproduct of Algorithm 1. Thus, we can decompose each part into up to $k$ monotone (increasing or decreasing, depending on whether the part is odd or even) subsequences. These subsequences can be then merged to obtain a sorted list $P_{\ell}$ of all elements in the corresponding part $S_{\ell}$ in $O(n \log k)$ overall time, for example by first merging pairs of subsequences, then quadruples (i.e., pairs of sequences which were obtained by merging pairs of original subsequences), and so on. Note that $P_{\ell}$ is increasingly ordered if $\ell$ is even, respectively decreasingly ordered if $\ell$ is odd.

\subsection{Combinatorial Lemmas}

Before moving on to the description of our algorithm, we state two important combinatorial observations, which relate an alternating $k$-decomposition to (longest) $k$-rollercoasters.

Proposition 1 Let $A$ be a k-rollercoaster in $S[1: n]$. Any part $S_{\ell}$ contains elements of at most four consecutive runs of $A$.

Proof By contradiction. Let $S_{\ell}^{\prime}$ be $S_{\ell}$ without the last element. If $S_{\ell}$ contains elements of five consecutive runs of $A$ then $S_{\ell}^{\prime}$ contains elements of four consecutive runs of $A$, and hence all elements of two such consecutive runs. Thus, if $S_{\ell}$ is an odd (even) part then $S_{\ell}^{\prime}$ contains LIS (LDS) of length $k$, which contradicts the definition of an alternating $k$-decomposition.

Lemma 2 Let $A$ be a longest $k$-rollercoaster in $S[1: n]$, and assume $x=S[j]$ is a nonfirst element occurring in an increasing run of A. Let $y=S\left[j^{\prime}\right]$ be the predecessor of $x$ in the respective increasing run of $A$, and consider an alternating $k$-decomposition of $S[1: n]$. Then, there exists $i$ such that $x$ is in $S_{i}$ and $y$ is in one of the parts $S_{i-4}, S_{i-3}, S_{i-2}, S_{i-1}, S_{i}$. 
Fig. 2 The increasing run $r$ from Lemma 2, with the points $x$ and $y$ highlighted

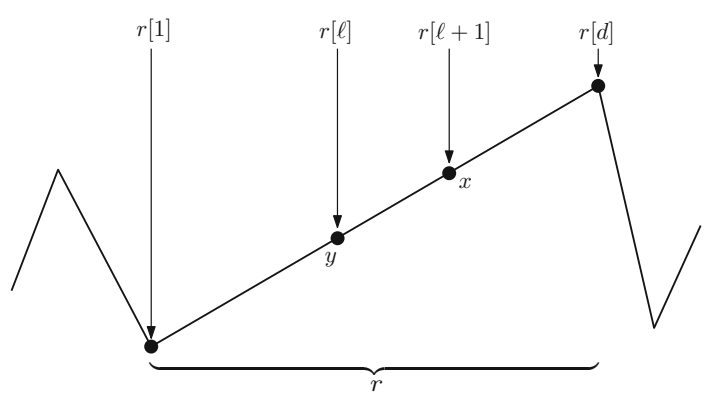

Proof By contradiction. Suppose that there are at least four parts between $x$ and $y$, i.e., $x$ is in $S_{i}$ and $y$ is in some $S_{k}$ with $k<i-4$. Let $r$ denote the run in the $k$-rollercoaster that contains $x$ and $y$, let $d \geq k$ be the length of $r$, and let $\ell$ be such that $r[\ell]=y$ and $r[\ell+1]=x$. By hypothesis, $r$ is an increasing run (see Fig. 2).

Consider the following four cases:

1. $\ell \leq k-1$ (i.e., there are at most $k-2$ elements in $r$ before $y$ ) and $k-2 \geq d-\ell-1$ (there are at most $k-2$ elements in $r$ after $x$ ).

2. $\ell \leq k-1$ and $k-1 \leq d-\ell-1$ (there at least $k-1$ elements in $r$ after $x$ ).

3. $\ell \geq k$ (there are at least $k-1$ elements in $r$ before $y$ ) and $k-2 \geq d-\ell-1$.

4. $\ell \geq k$ and $k-1 \leq d-\ell-1$.

Recall that there are at least four whole parts between $x$ and $y$. Therefore, in particular there are three consecutive parts $S_{i^{\prime}}, S_{i^{\prime}+1}$, and $S_{i^{\prime}+2}$ such that the first has LIS of length $k$, the second has LDS of length $k$, and the third has LIS of length $k$.

In the first case, we replace $r$ [2:d-1] with LIS of $S_{i^{\prime}}$, the LDS of $S_{i^{\prime}+1}$, and LIS of $S_{i^{\prime}+2}$. In this way, we clearly obtain a valid $k$-rollercoaster, and because we remove at most $2 k-4$ elements and add at least $3 k$, this creates a longer $k$-rollercoaster, which is a contradiction. In the second case, we replace $r[2: \ell]$ with LIS of $S_{i^{\prime}}$ and LDS of $S_{i^{\prime}+1}$. Again, we obtain a valid longer $k$-rollercoaster, because we remove at most $k-2$ elements and add at least $2 k$. Similarly, in the third case, we replace $r[\ell+1: d-1]$ with LDS of $S_{i^{\prime}+1}$ and LIS of $S_{i^{\prime}+2}$ to obtain a longer $k$-rollercoaster. Finally, in the fourth case we simply insert LDS of $S_{i^{\prime}+1}$ between $x$ and $y$ to obtain a longer $k$-rollercoaster.

In the above proof, we assumed that $r$, the run containing $x$, is an increasing run (as depicted in Fig. 2); the case when $r$ is decreasing can be treated in the same way and a similar conclusion is obtained.

\subsection{The Main Phase}

The description of this phase has, at its turn, several parts. First we give a general description of the idea behind our algorithm. Then we describe, at a high level, how this 
idea is implemented using dynamic programming. Finally, we give the full technical details.

The General Approach After the initial preprocessing phase we will compute a longest $k$-rollercoaster by dynamic programming.

For $1 \leq i \leq k$, we say that a subsequence of $S$ (not necessarily contiguous) is a $(k, i)_{+}$-rollercoaster if it ends with an increasing run of length exactly $i$ when $i<k$ and at least $k$ when $i=k$, while every other run is of length at least $k$. A $(k, i)_{-}$-rollercoaster is defined similarly, except that the last run should be decreasing. $(k, 1)_{+}$-rollercoasters can be seen as $(k, k)_{-}$-rollercoasters, while $(k, 1)_{-}-$ rollercoasters can be seen as $(k, k)_{+}$-rollercoasters. Thus, we can concentrate on $(k, i)_{+}-$and $(k, i)_{-}-$rollercoasters with $i \geq 2$.

The main idea in our approach is to construct, for every $2 \leq i \leq k$ and $1 \leq j \leq n$, a longest $(k, i)_{+}$-rollercoaster (respectively, $(k, i)_{-}$rollercoaster) ending with $S[j]$. To achieve this, we will calculate:

- $M_{+}[j, i]$ (respectively, $M_{-}[j, i]$ ), the position in $S$ of the predecessor of $S[j]$ in such a $(k, i)_{+}$-rollercoaster (respectively, $(k, i)_{-}$-rollercoaster), and

- $L_{+}[j, i]$ (respectively, $\left.L_{-}[j, i]\right)$, the length of the respective $(k, i)_{+}$-rollercoaster (respectively, $(k, i)_{-}$-rollercoaster).

We only describe in detail how to compute $M_{+}[j, i]$ and $L_{+}[j, i]$, as $M_{-}[j, i]$ and $L_{-}[j, i]$ are computed analogously. The main idea is that, by Lemma 2, we can restrict the search for $M_{+}[j, i]$ by using the alternating $k$-decomposition. If $S[j]$ is in the part $S_{\ell}$, then $M_{+}[j, i]$ should be in one of the parts $S_{\ell-4}, S_{\ell-3}, S_{\ell-2}, S_{\ell-1}$ or $S_{\ell}$. In each case, we compute a candidate for $M_{+}[j, i]$, as well as the length of the corresponding $(k, i)_{+}$-rollercoaster ending with $S[j]$. Finally, we store the predecessor of $S[j]$ on the longest such rollercoaster (from the possible candidates) and its length.

Consequently, in our computation, we will iterate, left to right, over the parts $S_{1}, S_{2}, \ldots$ of an alternating $k$-decomposition, and compute for each element $S[j]$ of the current part $S_{\ell}$ the values $M_{+}[j, i]$ and $L_{+}[j, i]$, for every $2 \leq i \leq k$. The following strategy covers all possibilities for the predecessor of $S[j]$ in a longest $(k, i)_{+}$-rollercoaster, so we can use the computed candidates to fill in the correct values of $M_{+}[j, i]$ of $L_{+}[j, i]$.

- For each $d \in\{1,2,3,4\}$ we will consider the elements $S[j]$ of $S_{\ell}$ in the order in which they appear in the ordered list $P_{\ell}$ and compute for each $S[j]$ in this list a respective candidate $M_{+}^{d}[j, i]$ for $M_{+}[j, i]$ : the position of the predecessor of $S[j]$ in a longest $(k, i)_{+}$-rollercoaster, assuming that the respective predecessor comes from $S_{\ell-d}$; the corresponding length $L_{+}^{d}[j, i]$ is also computed.

- Then, we compute another candidate $M_{+}^{\prime}[j, i]$ (and the corresponding length $\left.L_{+}^{\prime}[j, i]\right)$ by considering the case when the predecessor of $S[j]$ in a longest $(k, i)_{+}-$ rollercoaster comes from $S_{\ell}$. The order in which the elements of $S_{\ell}$ are considered in this case depends on whether $S_{\ell}$ has been decomposed into increasing or decreasing subsequences. 
The technical details of this approach are described gradually in the following paragraphs.

Dynamic Programming In this paragraph, we will describe in the main ideas of our dynamic programming approach, still without going into technicalities.

Let $S_{\ell}$ be a part of the alternating $k$-decomposition of $S$, as computed in the preprocessing phase. Assume that we want to compute the values stored in the arrays $M_{+}[\cdot, i], L_{+}[\cdot, i], M_{-}[\cdot, i]$ and $L_{-}[\cdot, i]$, for $2 \leq i \leq k$, corresponding to all elements $S[j]$ of the part $S_{\ell}$.

We assume that when we start computing these arrays for the part $S_{\ell}$, we have already computed $M_{+}\left[j^{\prime}, 1\right], M_{+}\left[j^{\prime}, 2\right], \ldots, M_{+}\left[j^{\prime}, k\right]$ and $L_{+}\left[j^{\prime}, 1\right], L_{+}\left[j^{\prime}, 2\right], \ldots$, $L_{+}\left[j^{\prime}, k\right]$, as well as $M_{-}\left[j^{\prime}, 1\right], M_{-}\left[j^{\prime}, 2\right], \ldots, M_{-}\left[j^{\prime}, k\right]$ and $L_{-}\left[j^{\prime}, 1\right], L_{-}\left[j^{\prime}, 2\right]$, $\ldots, L_{-}\left[j^{\prime}, k\right]$, for every element $S\left[j^{\prime}\right]$ of every part $S_{\ell^{\prime}}$ with $\ell^{\prime}<\ell$. The computation is split in several parts, which are first introduced at a high level, and then described precisely later.

Part 1 We will start with computing the candidates $M_{+}^{d}[j, i], L_{+}^{d}[j, i], M_{-}^{d}[j, i]$ and $L_{-}^{d}[j, i]$, but first for $2 \leq i<k$ only. The case $i=k$ will be handled separately, in Part 3 . These values correspond to the assumption that the predecessor $S\left[j^{\prime}\right]$ of $S[j]$ in the corresponding rollercoaster belongs to $S_{\ell-d}$, for some $d \in\{1,2,3,4\}$. Note that these candidates will be later used to set the correct values $M_{+}[j, i], L_{+}[j, i], M_{-}[j, i]$ and $L_{-}[j, i]$, for $1 \leq i \leq k$ and $S[j]$ an element of the part $S_{\ell}$. The main observation (and advantage) is that, in this case, the longest rollercoasters ending at $S\left[j^{\prime}\right]$ have been already correctly determined in our algorithm: it ends in a part $S_{\ell-d}$ and $\ell-d<\ell$. This makes the computation of the aforementioned candidates relatively simple.

Part 2 Then, we will consider the case that the predecessor $S\left[j^{\prime}\right]$ of $S[j]$ in the corresponding rollercoaster belongs to $S_{\ell}$, just like $S[j]$. We compute the candidates $M_{+}^{\prime}[j, i], L_{+}^{\prime}[j, i], M_{-}^{\prime}[j, i]$ and $L_{-}^{\prime}[j, i]$ for $M_{+}[j, i], L_{+}[j, i], M_{-}[j, i]$ and $L_{-}[j, i]$, respectively, again only for $2 \leq i<k$. Now, we need to be more careful in order to be able to guarantee that the longest rollercoaster ending at $S\left[j^{\prime}\right]$ is already known when $S[j]$ is considered. For this, we will proceed in several iterations. In the $t^{\text {th }}$ iteration, we will guarantee to compute the desired values under the restriction that only the last $t$ runs of the longest rollercoaster may contain elements from $S_{\ell}$ other than $S[j]$. It is worth noting that, as both $S[j]$ and its predecessor are assumed to be from $S_{\ell}$, the last run of the rollercoaster we search contains at least two elements from $S_{\ell}$. By Proposition 1, four iterations are enough in the above computation, as at most four runs of the longest $k$-rollercoaster can be in one part.

In each iteration, we start with setting, for all elements $S[g]$ of $S_{\ell}$, the initial candidates $M_{+}^{\prime}[g, 1], L_{+}^{\prime}[g, 1], M_{-}^{\prime}[g, 1]$ and $L_{-}^{\prime}[g, 1]$ corresponding to longest rollercoasters ending with $S[g]$, such that at most $t-1$ runs of these rollercoasters contain elements from $S_{\ell}$ other than $S[g]$. We handle the case of $S[g]$ being the only element in the rollercoaster by initially setting $L_{+}^{\prime}[g, 1]$ and $L_{-}^{\prime}[g, 1]$ to 1 . Otherwise, either $t=1$ and we need to extend longest rollercoasters ending with $S[g]$, such that the predecessor of $S[g]$ is not in $S_{\ell}$, or $t>1$ and we need to additionally consider longest rollercoasters ending with $S[g]$ computed in the previous iteration. 
Thus, we either copy the already known $L_{+}^{d}[g, k], M_{+}^{d}[g, k], L_{-}^{d}[g, k]$ and $M_{-}^{d}[g, k]$, with $d \in\{1,2,3,4\}$, or $L_{+}^{\prime}[g, k], M_{+}^{\prime}[g, k], L_{-}^{\prime}[g, k]$ and $M_{-}^{\prime}[g, k]$ calculated in the previous iteration.

In the $t$ th iteration, after the initialization we calculate (respectively, recalculate, if $t>1)$ the candidates $M_{+}^{\prime}[j, i], L_{+}^{\prime}[j, i], M_{-}^{\prime}[j, i]$ and $L_{-}^{\prime}[j, i]$, for $2 \leq i<k$, corresponding to the predecessor $S\left[j^{\prime}\right]$ of $S[j]$ in the last run of a longest $(k, i)_{+}$- or $(k, i)_{\text {- }}$-rollercoaster ending with $S[j]$, whose last $t$ runs only contain elements of $S_{\ell}$, belongs to the same part $S_{\ell}$. By performing the calculation for $i=2,3, \ldots, k-1$, in this order, we guarantee that the longest rollercoaster ending at the predecessor $S\left[j^{\prime}\right] \in S_{\ell}$, whose last $t$ runs only contain elements of $S_{\ell}$, and which ends with a run of length $i-1$, is already known. However, the computation is still not completely trivial and, as announced already, requires a different approach depending on whether $S_{\ell}$ was decomposed into at most $k$ increasing, respectively decreasing, subsequences.

Part 3 As announced, we need to handle the situation $i=k$ separately, as this also covers the case of the last run having more than $k$ elements. This, however, can be integrated in a rather straightforward way in our dynamic programming.

Finalizing the Computation Once we have identified for each $i$ and $S[j]$ of $S_{\ell}$ the candidates $M_{+}^{d}[j, i]$, for $d \in\{1,2,3,4\}$, and $M_{+}^{\prime}[j, i]$ for $M_{+}[j, i]$, and the corresponding lengths $L_{+}^{d}[j, i]$, for $d \in\{1,2,3,4\}$, and $L_{+}^{\prime}[j, i]$, which are candidates for $L_{+}[j, i]$, we will set $M_{+}[j, i]$ as the position $j$ which gives the maximum of the lengths $L_{+}^{d}[j, i]$, for $d \in\{1,2,3,4\}$, and $L_{+}^{\prime}[j, i]$, and set $L[j, i]$ accordingly.

Efficient Implementation We can now analyse in detail each part of our algorithm as cases introduced above, and discuss how they can be implemented efficiently. For the sake of easing the presentation, for each part, we first discuss how it is implemented (i.e., how the computation is conducted and which data structures are used and how) and then we compute the time complexity needed to perform the respective computation.

Part 1: Searching the Predecessor of $S[j]$ on a $(k, i)_{+}$-Rollercoaster in $S_{\ell-d}$ for Some $1 \leq d \leq 4$ We process $S_{\ell-d}$ to identify the candidates $M_{+}^{d}[j, i]$ and $L_{+}^{d}[j, i]$ for $M_{+}[j, i]$ and $L_{+}[j, i]$, respectively, for every $S[j] \in S_{\ell}$. The idea is to compute these candidates in the order in which the elements $S[j]$ occur in the sorted list $P_{\ell}$.

So, let us consider $P_{\ell}$ and $P_{\ell-d}$. For each element $S[j]$ in the current part (that is, in $\left.P_{\ell}\right)$ we want to identify a longest $(k, i-1)_{+}$-rollercoaster ending in $S_{\ell-d}$ with an element smaller or equal to $S[j]$. Thus, as $P_{\ell-d}$ is increasing, for every element $S[j]$ of the current part, from the list $P_{\ell}$, we need to consider only the elements in a prefix of $P_{\ell-d}$. Also, if $S\left[j^{\prime}\right]$ is to the right of $S[j]$ in $P_{\ell}$, that is, $S\left[j^{\prime}\right] \geq S[j]$, then the prefix of $P_{\ell-d}$ that we need to consider to compute $M_{+}^{d}\left[j^{\prime}, i\right]$ is at least as long as the prefix that we need to consider in order to compute $M_{+}^{d}[j, i]$. Therefore, we can use two pointers to sweep through $P_{\ell}$ and $P_{\ell-d}$ from left to right, and obtain the information needed to compute $M_{+}^{d}[j, i]$ and $L_{+}^{d}[j, i]$, for every $S[j] \in S_{\ell}$. At the beginning the pointers are positioned on the first element of $P_{\ell}$ and $P_{\ell-d}$, respectively. Say now, for a description of the general case, that the element currently pointed in 
$P_{\ell}$ and $P_{\ell-d}$ is $S[j]$ and $S[h]$, respectively (we update indices $j$ and $h$ along with the pointers). We keep moving forward the pointer corresponding to $S[h]$ until we find an element $S[h]>S[j]$. Then we set $M_{+}^{d}[j, i]=h^{\prime}$ and $L_{+}^{d}[j, i]=L_{+}\left[h^{\prime}, i\right]+1$, where $S\left[h^{\prime}\right]$ is an element occurring earlier than $S[h]$ in $P_{\ell-d}$ with the largest value of $L_{+}\left[h^{\prime}, i-1\right]$. The element $S\left[h^{\prime}\right]$ is maintained as we move from left to right in $P_{\ell-d}$. Then we proceed to the next element in $P_{\ell}$, i.e., advance the corresponding pointer one position in $P_{\ell}$.

Part 1: Complexity Using the implementation described above, computing candidates $M_{+}^{d}[j, i]$ and $L_{+}^{d}[j, i]$ for every $S[j] \in S_{\ell}$ takes, overall, $O\left(\left|S_{\ell-d}\right|+\left|S_{\ell}\right|\right)$ time.

Part 2, Initialization: Searching the Predecessor of $S[j]$ on a $(k, i)_{+}$-Rollercoaster in $S_{\ell}$ Recall that our process, in this case, works in four iterations. In the $t^{\text {th }}$ iteration, we will guarantee to compute the desired values under the restriction that only the last $t$ runs of the longest corresponding $(k, i)_{+}-$or $(k, i)_{-}$-rollercoaster may contain elements from $S_{\ell}$.

Let us consider the first iteration. We have already computed the values stored in the arrays $M_{+}\left[\cdot, i^{\prime}\right], M_{-}\left[\cdot, i^{\prime}\right], L_{+}\left[\cdot, i^{\prime}\right], L_{-}\left[\cdot, i^{\prime}\right]$ for all elements occurring in parts $S_{\ell}^{\prime}$ with $\ell^{\prime}<\ell$. So, we can set the initial values $L_{+}^{\prime}[j, 1]$ (respectively, $L_{-}^{\prime}[j, 1]$ ) corresponding to $S[j]$ being the first element of its increasing (respectively, decreasing) run. These were calculated in Case 1 , when we identified longest $(k, k)_{-}$-rollercoasters (respectively, $(k, k)_{+}$-rollercoasters), whose last run ends with $S[j]$ preceded by an element of $S_{\ell-d}$, with $d \in\{1, \ldots, 4\}$. If no longest $(k, k)_{-}$-rollercoaster (respectively, $(k, k)_{+}$-rollercoaster) ending in $S[j]$ was identified in Case 1 , we simply start a new rollercoaster of length 1 with $j$ being its single element. Then we can proceed with the calculation for $i=2,3, \ldots, k-1$, as in the case of later iterations.

For later iterations $t=2,3,4$, the initial values $L_{+}^{\prime}[j, 1]$ (respectively, $L_{-}^{\prime}[j, 1]$ ) corresponding to $S[j]$ being the first element of its increasing (respectively, decreasing) run were calculated already, when longest $(k, k)_{-}$-rollercoasters (respectively, $(k, k)_{+}$-rollercoasters) with $t-1$ runs containing elements of $S_{\ell}$ were identified.

We now proceed to describing how to compute the candidate in the $t^{\text {th }}$ iteration. The goal is to identify candidates, denoted $M_{+}^{\prime}[j, i]$ and $L_{+}^{\prime}[j, i]$ (for $M_{+}[j, i]$ and $L_{+}[j, i]$, respectively) for every $S[j] \in S_{\ell}$ and $i=2,3, \ldots, k-1$. By appropriately ordering the computation, when considering $S[j]$ and $i$ we can assume that we have already computed the predecessor of each $S[g]$ in (as well as the length of) a longest $\left(k, i^{\prime}\right)_{+}$-rollercoaster with at most $t$ runs containing elements from $S_{\ell}$ other than $S[g]$, for every $i^{\prime}<i$ and every $S[g] \in S_{\ell}$. Computing $M_{-}^{\prime}[j, i]$ and $L_{-}^{\prime}[j, i]$ is symmetric.

Part 2, Case 1: Searching the Predecessor of $S[j]$ on a $(k, i)_{+}$-Rollercoaster in $S_{\ell}$, Which was Decomposed Into $k$ Increasing Subsequences Consider the decomposition of $S_{\ell}$ into $k$ increasing subsequences $I_{1}, I_{2}, \ldots, I_{k}$. The elements of every sequence are increasing w.r.t. their values and w.r.t their positions in $S$. Consider an element $S[j] \in I_{a}$ and $1 \leq b \leq k$ (possibly $a=b$ ). The elements of $I_{b}$ that can be the predecessor of $S[j]$ in a $(k, i)_{+}$-rollercoaster (that is, possible choices for $M_{+}^{\prime}[j, i]$ ) are both less than $S[j]$ w.r.t. value and w.r.t. position in $S$. Thus, these elements form a prefix of $I_{b}$. Moreover, for every $S[j] \in I_{a}$ and $1 \leq b \leq k$ we want to maximise 
$L_{+}^{\prime}\left[h^{\prime}, i\right]$ over all elements $S\left[h^{\prime}\right]$ in such a prefix. Also, just as in the previous case, if we process the elements of $I_{a}$ in the order in which they occur in $I_{a}$, we will have that the prefix that corresponds to $S\left[j^{\prime}\right]$ is longer than the prefix that corresponds to $S[j]$, if $S\left[j^{\prime}\right]$ occurs after $S[j]$ in $I_{a}$. Therefore, we can again use two pointers to sweep through $I_{a}$ and $I_{b}$ and compute, for every $S[j] \in I_{a}$, the element $S\left[h^{\prime}\right] \in I_{b}$ that could precede $S[j]$ in a $(k, i)_{+}$-rollercoaster with the largest value of $L_{+}^{\prime}\left[h^{\prime}, i-1\right]$. Finally, we set $M_{+}^{\prime}[j, i]$ and $L_{+}^{\prime}[j, i]$ to be the position $h^{\prime}$, and, respectively, the length $\left.L_{[}^{\prime} h^{\prime}, i-1\right]+1$, which correspond to the largest such value $L_{+}^{\prime}\left[h^{\prime}, i-1\right]$ among all $S\left[h^{\prime}\right]$ in the runs $I_{b}$ with $1 \leq b \leq k$.

Part 2, Case 1: Complexity For a fixed $a$, the procedure described in the paragraph above takes $O\left(k\left|I_{a}\right|+\sum_{1 \leq b \leq k}\left|I_{b}\right|\right)=O\left(k\left|I_{a}\right|+\left|S_{\ell}\right|\right)$ time. Adding everything up, computing the candidates $\bar{M}_{+}^{\prime}[j, i]$ and $L_{+}^{\prime}[j, i]$ for every $S[j] \in S_{\ell}$ takes $O\left(k\left|S_{\ell}\right|\right)$ time.

Part 2, Case 2: Searching the Predecessor of $S[j]$ on a $(k, i)_{+}$-Rollercoaster in $S_{\ell}$, Which was Decomposed into $k$ Decreasing Subsequences This is the most complicated case. Recall that the decomposition into $k$ decreasing subsequences $D_{1}, D_{2}, \ldots, D_{k}$ was obtained with Algorithm 1. In more detail, $D_{a}$ consists of elements assigned to $R[a]$ throughout the execution of the algorithm. Thus, if $S[j] \in D_{a}$ then the predecessor of $S[j]$ in a sought longest $(k, i)_{+}$-rollercoaster, denoted $S\left[j^{\prime}\right]$, must belong to $D_{b}$ for some $1 \leq b<a$. Indeed, Algorithm 1 first processes $S\left[j^{\prime}\right]$ and then $S[j]$, so if $S\left[j^{\prime}\right] \in D_{b}$ then $R[b] \leq S\left[j^{\prime}\right]$ when processing $S[j]$ and consequently $S\left[j^{\prime}\right]<S[j]$ implies that $S[j]$ is assigned to $R[a]$ with $a>b$. So, we first compute the candidates $M_{+}^{\prime}[j, i]$ and $L_{+}^{\prime}[j, i]$ for every $S[j] \in D_{1}$, then for every $S[j] \in D_{2}$, and so on.

Consider a decreasing subsequence $D_{a}$ and suppose that we have already processed all elements in $D_{1}, D_{2}, \ldots, D_{a-1}$. Note that at this point we have already computed, for every $S\left[j^{\prime}\right] \in D_{1} \cup \cdots \cup D_{a-1}$, the values of $M_{+}^{d}\left[j^{\prime}, i\right]$ and $L_{+}^{d}\left[j^{\prime}, i\right]$, for $1 \leq d \leq 4$, as well as the values $M_{+}^{\prime}\left[j^{\prime}, i\right]$ and $L_{+}^{\prime}\left[j^{\prime}, i\right]$ corresponding to the current iteration. Thus, we have already correctly updated $M_{+}\left[j^{\prime}, i\right]$ and $L_{+}\left[j^{\prime}, i\right]$ by choosing the option that maximises the length of the corresponding $(k, i)_{+}$-rollercoaster, which is important for the case of $i=k$.

Consider now an element $S[j] \in D_{a}$ and $1 \leq b<a$. The elements of $D_{b}$ that can be the predecessor of $S[j]$ in a $(k, i)_{+}$-rollercoaster (that is, possible candidates for $\left.M_{+}^{\prime}[j, i]\right)$ are both less w.r.t. value and w.r.t. position in $S$, similarly to the previous case. The difference is that now these elements form contiguous subsequence $X$ of $D_{b}$ that is not necessarily a prefix. The first element of $X$ can be found by searching for the first element with sufficiently small value, while its last element can be found by searching the last element with sufficiently small position (note that $X$ might be empty). Let $S[h]$ be the next element after $S[j]$ in $D_{a}$, and $Y$ be its corresponding contiguous subsequence of $D_{b}$ consisting of possible predecessors in a $(k, i)_{+}$-rollercoaster. Clearly, $S[j]>S[h]$ while $j<h$. Thus, the first element of $Y$ is either the same as the first element of $X$ or occurs after the first element of $X$ in $D_{a}$, while the last element of $Y$ is either the same as the last element of $X$ or occurs after the last element of $X$ in $D_{a}$ (assuming that both $X$ and $Y$ are non-empty). Thus, 
we sweep through $D_{a}$ while maintaining the current contiguous subsequence $X$ of $D_{b}$ corresponding to the possible predecessors of the current $S[j] \in D_{a}$. This requires the following tool.

Lemma 3 ([13]) There is a data structure that maintains a list of elements under the following operations: pop an element from the front, push an element in the back, and return the maximum element in the current list, each in $O$ (1) time.

When processing the current element $S[j] \in D_{a}$ we maintain the first element $S[f] \in D_{b}$ such that $S[f]<S[j]$ and the last element $S[\ell] \in D_{b}$ such that $\ell<j$. Then $X$ consists of all elements between $S[f]$ and $S[\ell]$ in $D_{b}$ (inclusive), and is maintained in a structure from Lemma 3 storing the lengths of their corresponding $(k, i)_{+}$-rollercoaster, that is, the already known value of $L_{+}[\cdot, i-1]$. This allows us to extract the element $S\left[j^{\prime}\right] \in X$ with the largest value of $L_{+}\left[j^{\prime}, i-1\right]$, and set $M_{+}^{\prime}[j, i]=j^{\prime}$ and $L_{+}^{\prime}[j, i]=L_{+}\left[j^{\prime}, i-1\right]+1$ in constant time, while updating $f$ and $\ell$ takes amortised constant time.

Part 2, Case 2: Complexity We can now conclude that, based on Lemma 3 and the explanation following it, that, as in the previous case, for a fixed $a$, this procedure takes $O\left(k\left|D_{a}\right|+\sum_{1 \leq b \leq k}\left|D_{a}\right|\right)=O\left(k\left|D_{a}\right|+\left|S_{\ell}\right|\right)$ time, so $O\left(k\left|S_{\ell}\right|\right)$ overall.

Part 3: Choosing the Best Candidate for $M_{+}[i, j]$ and $L_{+}[i, j]$ Once we have computed, for some $i<k$, all the candidates $M_{+}^{d}[j, i]$, with $d \in\{1,2,3,4\}$, and $M_{+}^{\prime}[j, i]$ for $M_{+}[j, i]$ and $L_{+}^{d}[j . i]$, with $d \in\{1,2,3,4\}$, and $L_{+}^{\prime}[j, i]$ for $L_{+}[j, i]$, we can see which pair of candidates corresponds to the longest $(k, i)_{+}$-rollercoaster ending with $S[j]$, and set $M_{+}[j, i]$ and $L_{+}[j, i]$ accordingly. The case of $(k, i)_{-}$-rollercoasters is treated identically.

Part 3: How to Deal With $i=k$ To compute candidates for $M_{+}[j, k]$ and $L_{+}[j, k]$, we first use exactly the same dynamic programming approach as before (in each respective case) for $i=k$. This means that we use the values computed for $M_{+}[\cdot, k-1]$ and $L_{+}[\cdot, k-1]$. But this only allows us to compute the length of a longest $(k, k)_{+^{-}}$ rollercoaster with the last run of length exactly $k$. To extend this to arbitrary $(k, k)_{+}-$ rollercoasters with the last run of length greater than $k$ we additionally run the same algorithm but, instead of looking at $M_{+}[\cdot, k-1]$ and $L_{+}[\cdot, k-1]$, we use in our dynamic programming also the already computed values/candidates for $M_{+}[\cdot, k]$ and $L_{+}[\cdot, k]$. More precisely, the values we already computed from these arrays are treated in this extra step exactly as in the cases described above. The reason why this works is that, due to the order in which we consider the elements of $S_{\ell}$, at the moment when we compute the length of a longest $(k, k)_{+}$-rollercoaster ending with $S[j]$, and which may have more than $k$ elements in the final run, we have already computed (in each respective case) the length of a longest $(k, k)_{+}$-rollercoaster ending with any element $S\left[j^{\prime}\right]$ which may be a predecessor of $S[j]$ on the respective $(k, k)_{+}$-rollercoaster.

Conclusion and Overall Complexity With these final remarks, our algorithm is completely described. It only remains to find the element $S[j]$ for which $\max \left\{L_{-}[j, k], L_{+}[j, k]\right\}$ is maximum. The correctness follows from the comments made throughout its description. To compute the complexity, it is enough to note that 


\begin{tabular}{|c|c|c|c|c|}
\hline 0 & 1 & 2 & 2 & 2 \\
\hline-1 & 0 & 1 & 1 & 2 \\
\hline-2 & -1 & 0 & 1 & 2 \\
\hline-3 & -2 & -1 & 0 & 1 \\
\hline-4 & -3 & -2 & -1 & 0 \\
\hline
\end{tabular}
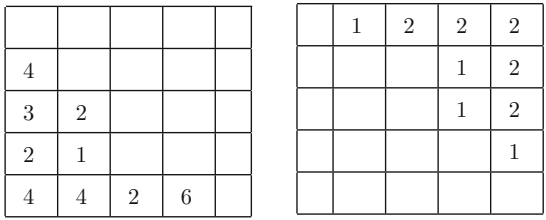

Fig. 3 Anti-Monge matrix, reverse falling staircase anti-Monge matrix, and falling staircase anti-Monge matrix

each part $S_{\ell}$ of the partition of $S$ is processed in $O\left(k\left|S_{\ell}\right|\right)$ time, for each $1 \leq i \leq k$. Adding this up, the total complexity of our algorithm is $O\left(n k^{2}\right)$, and we have shown the following.

Theorem 3 For every sequence $S[1: n]$ and $k \geq 3$, the length of a longest $k$ rollercoaster in $S$ can be found in $O\left(n k^{2}\right)$-time.

\section{Computing a Longest $k$-Rollercoaster in $O\left(n \log ^{2} n\right)$-Time}

Before we describe our algorithm, we introduce two preliminary procedures. Firstly, we introduce the definition of an anti-Monge matrix and the algorithm for finding the maximum in every column of such a matrix. Secondly, we describe the algorithm for finding LIS in contiguous subsequences of the input sequence. Finally, we describe the algorithm computing a longest $k$-rollercoaster in this sequence, using the previously developed tools as black boxes.

Monge Matrices Let $A$ be an $n \times n$ matrix, and $A[i, j]$ denote its element in the $i^{\text {th }}$ row from the top and the $j^{\text {th }}$ column from the left. $A$ is Monge (respectively, antiMonge) if, for every $1 \leq i<j \leq n$ and $1 \leq k<\ell \leq n$, the Monge equality holds, namely $A[i, k]+A[j, \ell] \leq A[i, \ell]+A[j, k]$ (respectively, $A[i, k]+A[j, \ell] \geq$ $A[i, \ell]+A[j, k])$. An $n \times n$ falling staircase anti-Monge matrix is a matrix with blanks such that for every blank all elements below and to the left are blanks, and the anti-Monge inequality holds whenever the four concerned elements are non-blank. Similarly, an $n \times n$ reverse falling staircase anti-Monge matrix is a matrix with blanks such that for every blank all elements above and to the right are blanks, and the antiMonge inequality holds whenever the four concerned elements are non-blank. Finally, an $n \times n$ matrix $A$ is totally monotone if, for every $1 \leq i<j \leq n$ and $1 \leq k<\ell \leq n$, $A[i, k] \leq A[i, \ell]$ implies $A[j, k] \leq A[j, \ell]$.

Let us now recall some basic facts regarding Monge matrices.

Observation 1 Adding the same value to every element in a row (or a column) of an anti-Monge matrix results in another anti-Monge matrix.

Observation 2 To check if a matrix is anti-Monge it is sufficient to check if every contiguous $2 \times 2$ submatrix is anti-Monge.

Looking at the first matrix in Fig. 3, we can see that it is anti-Monge because in every contiguous $2 \times 2$ submatrix the sum of the elements on the main-diagonal is greater or equal to the sum of the elements on the anti-diagonal. 
Fig. 4 A permutation matrix $A$ and its distribution matrix $A^{\Sigma}$

\begin{tabular}{|l|l|l|}
\hline 0 & 1 & 0 \\
\hline 1 & 0 & 0 \\
\hline 0 & 0 & 1 \\
\hline
\end{tabular}

\begin{tabular}{|l|l|l|l|}
\hline 0 & 1 & 2 & 3 \\
\hline 0 & 1 & 1 & 2 \\
\hline 0 & 0 & 0 & 1 \\
\hline 0 & 0 & 0 & 0 \\
\hline
\end{tabular}

The following lemma follows from the well-known SMAWK algorithm [2].

Lemma 4 (Lemma 3.3 in Aggarwal et al. [1]) The maximal elements in all rows (i.e., all row maxima) of a reverse falling staircase totally monotone matrix can be found in $O(n)$ time.

By transposing the matrix and observing that being anti-Monge implies being totally monotone we obtain the following.

Corollary 1 The maximal elements in all columns (i.e., all column maxima) of a falling staircase anti-Monge matrix can be found in $O(n)$ time.

LIS-in-Range Queries Let $S[1: n]$ be the input sequence. Define $M$ as an $(n+1) \times$ $(n+1)$ matrix with 0 -indexed rows and columns, such that $M[i, j]$ is the length of LIS in $S[i+1: j]$ for $i<j$ and $M[i, j]=j-i$ otherwise (the anti-Monge matrix in Fig. 3 is such a matrix for the sequence $(3,4,1,2)$ ). As hinted by our example, this matrix turns out to have a rather special structure as observed by Tiskin [21]. We describe this structure in the following.

Let $S^{\prime}$ be the sequence obtained by sorting $S$ (recall that $S$ consists of distinct elements), and observe that LIS of $S$ is the same as a longest common subsequence (LCS, for short) of $S$ and $S^{\prime}$. Thus, we can think that $M[i, j]$ is LCS of $S^{\prime}$ and $S[i+1: j]$. As such, the following result can be shown (see [21] and the references therein).

\section{Lemma 5 is anti-Monge.}

Our algorithm needs to access the elements of $M$. Since the matrix contains $(n+1)^{2}$ elements, it is too large to be explicitly stored in memory. Fortunately, Tiskin also showed how to create in $O\left(n \log ^{2} n\right)$ time an $O(n)$-space implicit representation of $M$ that allows us to obtain any of its elements in $O(\log n)$ time [21]. Before we present the internals of this representation, we need to introduce some additional definitions illustrated in Fig. 4.

Definition 1 Let $A$ be any $n \times n$ matrix. Its distribution matrix $A^{\Sigma}$ is an $(n+1) \times(n+1)$ matrix defined by $A^{\Sigma}[x, y]=\sum_{i \geq x, j<y} A[i, j]$, for every $1 \leq x \leq n+1,1 \leq y \leq$ $n+1$.

Definition 2 A permutation matrix is a square matrix that has exactly one 1 in every row and column, and the remaining elements are equal to 0 .

Now, we can provide the final ingredients of the construction. For two strings $w_{1}$ and $w_{2}$ of length $d$, Tiskin defines in [21] a $(2 d+1) \times(2 d+1)$ matrix $L$ in the following 
way. Let $w_{2}^{\prime}$ be the string equal to $?^{d} w_{2} ?^{d}$, whose positions are indexed from $-(d-1)$ to $2 d$. The rows of $L$ are indexed from $-d$ to $d$, while the columns of $L$ are indexed from 0 to $2 d$. The elements of $L$ are defined by $L[i, j]=\operatorname{LCS}\left(w_{1}, w_{2}^{\prime}[i+1: j]\right)$ if $j>i$, and $L[i, j]=j-i$ otherwise. In this definition, it is assumed that ? matches any character. If $w_{2}$ is the input sequence $S$ and $w_{1}$ is $S^{\prime}$ then, for $0 \leq i, j \leq n$ we have $L[i, j]=M[i+1, j+1]$. Tiskin proved (Theorem 4.10 in [21]) that there exists $2 d \times 2 d$ permutation matrix $P$ such that $L[i, j]=j-i-P^{\Sigma}[i, j]$. Furthermore, he provided an $O\left(n \log ^{2} n\right)$-time algorithm that finds all the non-zero entries of $P$ (Algorithm 8.2 in [21]). Having all the non-zero entries of $P$ we can apply a dominance counting structure of Chazelle [8] that can be constructed in $O(n \log n)$ time, uses $O(n)$ space, and calculates $P^{\Sigma}[i, j]$ and hence also $M[i+1, j+1]$ in $O(\log n)$ time. Summarising, in $O\left(n \log ^{2} n\right)$ time we obtain a structure that returns any element of $M$ in $O(\log n)$ time. We similarly obtain a matrix storing the length of LDS of every $S[i+1: j]$.

\section{Description of the Algorithm}

Let $S[1: n]$ be the input sequence. For every $1 \leq x \leq n$, let res $[x]$ be the length of a longest $k$-rollercoaster in $S[1: x]$, and inc $[x]$ (respectively, $\operatorname{dec}[x]$ ) be the length of a longest $k$-rollercoaster in $S[1: x]$ with the last run increasing (respectively, decreasing). Note that we do not require that these $k$-rollercoasters contain $S[x]$. Then, $\operatorname{res}[x]=\max \{\operatorname{dec}[x], \operatorname{inc}[x]\}$, for $1 \leq x \leq n$. Firstly, we introduce two structural lemmas.

Lemma 6 Let $A$ be a k-rollercoaster in $S[1: i]$ with the last run decreasing, and $r$ be an increasing subsequence in $S[i: n]$ such that $|r| \geq k$. Then there exists a $k$-rollercoaster in $S[1: n]$ of length at least $|A|+|r|-1$ with the last run increasing.

Proof Let $A^{\prime}$ be the sequence consisting of all elements from both $A$ and $r$. Recall that a sequence is a $k$-rollercoaster if every run has length at least $k$. In order to show that $A^{\prime}$ is a $k$-rollercoaster with last run increasing we need to consider three cases: the first element of $r$ is the last element of $A$, the first element of $r$ is greater than the last element of $A$, and the first element of $r$ is less than the last element of $A$.

In the first case, all runs in $A^{\prime}$ but the last are the same as in $A$, and the last run is equal to $r$. Since $A$ is a $k$-rollercoaster and $|r| \geq k$ we conclude that $A^{\prime}$ is a $k$-rollercoaster. $A$ and $r$ have one common element, so $\left|A^{\prime}\right|=|A|+|r|-1$.

In the second case, all runs in $A^{\prime}$ but the last are also the same as in $A$, and the last run consists of the last element of $A$ and $r$. Again we conclude that $A^{\prime}$ is a $k$-rollercoaster. Since $A$ and $r$ have no common elements, $\left|A^{\prime}\right|=|A|+|r|$.

In the third case, all runs in $A^{\prime}$ but the last two are the same as in $A$. The secondto-last run in $A^{\prime}$ consists of the last run of $A$ and the first element of $r$, and the last run in $A^{\prime}$ is $r$. Hence, $A^{\prime}$ is a $k$-rollercoaster. Since $A$ and $r$ have no common elements, $\left|A^{\prime}\right|=|A|+|r|$.

Lemma 7 Consider a longest $k$-rollercoaster in $S[1: n]$ with the last run increasing (respectively, decreasing), and let $r$ be its last run with the first element $S[i]$. Then $r$ is a longest increasing (respectively, decreasing) subsequence in $S[i: n]$. 
Proof The case when the longest $k$-rollercoaster in $S[1: n]$, with the last run increasing, consists of a single run is immediate: we can replace that run by a longer one, e.g., the LIS of $S[i: n]$, and obtain a longer $k$-rollercoaster.

So, let us assume that the longest $k$-rollercoaster in $S[1: n]$, with the last run increasing, has at least 2 runs. The proof follows by contradiction. Let $A$ be a longest $k$-rollercoaster from the statement of the lemma, and suppose that there exists a longer increasing sequence $r^{\prime}$ in $S[i: n]$. That is, $\left|r^{\prime}\right|>|r|$. Let $A^{\prime}$ be the prefix of $A$ ending at $S[i]$ (clearly, $\left|A^{\prime}\right| \leq k$ ). Observe that $\left|A^{\prime}\right|=|A|-|r|+1$. Then by Lemma 6 there exists a $k$-rollercoaster in $S$ of length at least $\left|A^{\prime}\right|+\left|r^{\prime}\right|-1=|A|-|r|+\left|r^{\prime}\right|>|A|$.

The above lemmas allow us to obtain the formula for calculating the arrays inc and dec. Recall that $M[i, j]$ is the length of LIS in $S[i+1: j]$. Let $M^{\prime}$ be the matrix obtained from $M$ by replacing all elements less than $k$ by $-\infty$, and let $Z\left(j, j^{\prime}\right)$ be the set of indices $j \leq i \leq j^{\prime}$ such that length of LIS in $S\left[i: j^{\prime}\right]$ is at least $k$ (or, in other words, $\left.M^{\prime}\left[i-1, j^{\prime}\right] \neq-\infty\right)$.

Proposition 2 For every $1 \leq x \leq n$, the following holds:

$$
\begin{aligned}
\operatorname{inc}^{\prime}[x] & =\max \left\{\operatorname{dec}[i]+M^{\prime}[i-1, x]-1: i \in Z(1, x)\right\}, \\
\operatorname{inc}[x] & =\max \left\{\operatorname{inc}^{\prime}[x], M^{\prime}[0, x]\right\} .
\end{aligned}
$$

If $Z(1, x)$ is empty then we set inc ${ }^{\prime}[x]=0$.

Proof By Lemma 6 we obtain that for every $i \in Z(1, x)$ there exists a $k$-rollercoaster in $S[1: x]$ with the last run increasing of length at least $\operatorname{dec}[i]+M^{\prime}[i-1, x]-1$. We conclude that inc ${ }^{\prime}[x]$ is less or equal to the length of a longest $k$-rollercoaster with the last run increasing in $S[1: x]$. Observe that $M^{\prime}[0, x]$ corresponds to an increasing run of length at least $k$ or is equal to $-\infty$. We obtain that inc $[x]$ is less or equal than the length of a longest $k$-rollercoaster with the last run increasing in $S[1: x]$.

For the converse, consider a $k$-rollercoaster $A$ with the last run increasing in $S[1: x]$. If $A$ consists of just a single run then its length is $M^{\prime}[0, x]$. Otherwise, let $S[i]$ be the first element in the last run of $A$. Then by Lemma 7 the length of the last run is equal to $M^{\prime}[i-1, x]$ and the length of $A$ is $\operatorname{dec}[i]+M^{\prime}[i-1, x]-1$. Overall, the length of $A$ is at most inc $[x]$.

Proposition 2 cannot be applied directly if we aim to achieve the announced $O\left(n \log ^{2} n\right)$ time complexity, and we need to introduce some auxiliary definitions. For every $1 \leq d \leq x$ we define inc $d[x]$ as follows:

$$
\begin{aligned}
\operatorname{inc}_{d}^{\prime}[x] & =\max \left\{\operatorname{dec}[i]+M^{\prime}[i-1, x]-1: i \in Z(1, d-1)\right\}, \\
\text { inc }_{d}[x] & =\max \left\{\operatorname{inc}_{d}^{\prime}[x], M^{\prime}[0, x]\right\} .
\end{aligned}
$$

If $Z(1, d-1)$ is empty then we set inc ${ }_{d}^{\prime}[x]=0$. In other words, inc ${ }_{d}[x]$ is equal to the length of a longest $k$-rollercoaster in $S[1: x]$ with the last run increasing and starting at an element $S[i]$ with $i<d$ or LIS of $S[1: x]$ of length at least $k$. Thus, inc ${ }_{1}[x]$ is equal to either 0 or the length of a LIS in $S[1: x]$. We similarly define $\operatorname{dec}_{d}[x]$. 
Observation 3 For every $j>i-k+1$ inc $_{j}[i]=$ inc $[i]$.

We describe a function COMPUTE that receives a contiguous subsequence $S[i: j]$ together with the previously calculated arrays $\operatorname{inc}_{i}[i: j]$ and $\operatorname{dec}_{i}[i: j]$, and returns the arrays inc $[i: j]$ and $\operatorname{dec}[i: j]$. To calculate the length of a longest $k$-rollercoaster in $S[1: n]$ we invoke the function with the whole $S[1: n]$ and the arrays inc ${ }_{1}[1: n]$, $\operatorname{dec}_{1}[1: n]$ as arguments, and return the maximum over the two resulting arrays. Note that inc $c_{1}[1: n]$ and $\operatorname{dec}_{1}[1: n]$ can be calculated in $O(n \log n)$ time using Algorithm 1.

Let $m=\left\lceil\frac{i+j}{2}\right\rceil$. The main idea of COMPUTE is to call the function recursively for the left half to calculate inc $[i: m-1]$ and $\operatorname{dec}[i: m-1]$. The next step is to calculate inc $_{m}[m: j]$ and $\operatorname{dec}_{m}[m: j]$ using tools from the previous paragraphs (as described below). Finally, we recursively calculate inc $[m: j]$ and $\operatorname{dec}[m: j]$. Concatenating the results from both recursive calls gives us the desired result. This is summarised in Algorithm 2.

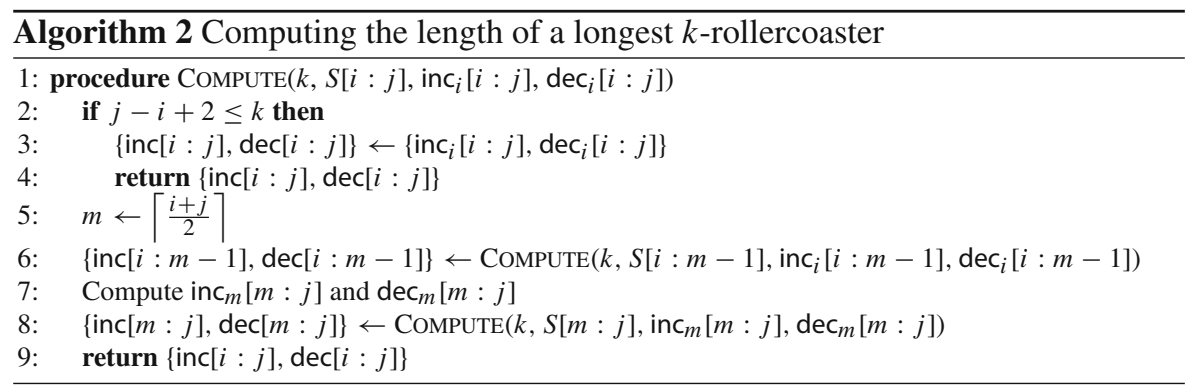

Computing inc $m[m: j]$ and $\operatorname{dec}_{m}[m: j]$ We only describe how to calculate inc $m[m$ : $j]$, as $\operatorname{dec}_{m}[m: j]$ can be computed by a similar approach. Recall the previously introduced matrix $M^{\prime}$, obtained by replacing values less than $k$ by $-\infty$ in $M$. Let $A_{\text {inc }}$ be the $(m-i) \times(j+1-m)$ matrix with rows indexed from $i$ to $m-1$ and columns indexed from $m$ to $j$ satisfying:

$$
A_{\text {inc }}[x, y]= \begin{cases}\operatorname{dec}[x]+M^{\prime}[x-1, y]-1 & \text { when } M^{\prime}[x-1, y] \neq-\infty \\ \text { blank } & \text { otherwise. }\end{cases}
$$

Since we are able to retrieve any element of $M^{\prime}$ in $O(\log n)$ time using LIS-in-range queries, and the value of $\operatorname{dec}[x]$, for every $i \leq x \leq m-1$, is already available, each element of $A_{\text {inc }}$ can be calculated in $O(\log n)$ time. Furthermore, we have the following property.

Proposition 3 A is a falling staircase anti-Monge matrix.

Proof By Lemma $5 M$ is an anti-Monge matrix. By Observation 1 this is still the case if we add the same value to all elements in the same row. 
To prove that $A$ is a falling staircase matrix consider a non-blank element $A[i, j]$. Then $M[i, j] \geq k$. But this implies $M[i-1, j] \geq k$ and $M[i, j+1] \geq k$ (as long as $i>1$ and $j<n$ ), so all elements above and to the right are also non-blank as required.

Proposition 4 For every $m \leq \ell \leq j$, inc $c_{m}[\ell]$ is equal to either inc $c_{i}[\ell]$ or the maximum in the $\ell^{\text {th }}$ column of $A$.

Proof For every $m \leq \ell \leq j$, inc $m[\ell]$ is equal to either inc ${ }_{i}[\ell]$ or $\max \left\{\operatorname{dec}[j]+M^{\prime}[j-\right.$ $1, \ell]-1: j \in Z(i, m-1)\}$. However, the latter is exactly the maximum in the $\ell^{\text {th }}$ column of $A$.

Lemma 8 We can compute inc ${ }_{m}[m: j]$ and $\operatorname{dec}_{m}[m: j]$ in $O((j-i+1) \log n)$ time.

Proof By Proposition 4 computing inc $m[m: j]$ reduces to finding all the column maxima in $A$. Since $A$ is a falling staircase anti-Monge matrix, we can use the algorithm from Corollary 1 . Access to any element of $A$ requires $O(\log n)$ time, so in total we obtain $O((j-i+1) \log n)$ time complexity.

We can now state with the main result of this section.

Theorem 4 For every sequence $S[1: n]$ and $k \geq 3$, the length of a longest $k$ rollercoaster in $S$ can be found in $O\left(n \log ^{2} n\right)$ time.

Proof The algorithm needs $O\left(n \log ^{2} n\right)$ preprocessing time to construct the LIS-inrange (and LDS-in-range) structure. We compute inc ${ }_{1}[1: n]$ and $\operatorname{dec}_{1}[1: n]$ in $O(n \log n)$ time using Algorithm 1. Then, we call the recursive function COMPUTE. By Lemma 8 a call of the function on $S[i: j]$ takes $O((j-i+1) \log n)$ time, so its running time is described by the recurrence $T(n)=2 T(n / 2)+O(n \log n)$ that solves to $O\left(n \log ^{2} n\right)$. Thus, the overall time complexity is $O\left(n \log ^{2} n\right)$.

\section{Constructing a Longest $\boldsymbol{k}$-Rollercoaster}

In this section we briefly discuss how to construct a longest rollercoaster for both algorithms.

For the $O\left(n k^{2}\right)$ Algorithm In the respective algorithm, for each $2 \leq i \leq k$, and for each element $S[j]$, we compute the predecessor of $S[j]$ on a longest (not necessarily contiguous) subsequence of $S$ ending with $S[j]$ and with every run of length at least $k$, except for the last run, which has only $i$ elements if $i<k$ and at least $k$ elements if $i=k$. If, together with this predecessor, we store also the length of the last run in the respective subsequence of $S$, we can trace the whole sequence back. Indeed, the predecessor gives us the information what element should we list before $S[j]$ in the subsequence. The length of the run gives us information on the length of the run ending with the predecessor of $S[j]$, so we know where we should look in our data structures for the predecessor of $S[j]$. For some $i$ and $j$, tracing back a longest (not necessarily contiguous) subsequence of $S$ ending with $S[j]$ and with every run of length at least 
$k$, except for the last run, which has only $i$ elements if $i<k$ and at least $k$ elements if $i=k$, takes, clearly, $O(n)$ time, provided that we have the information described above.

In the end, we will only need to trace back a longest (not necessarily contiguous) subsequence of $S$ ending with some element $S[j]$ and with every run of length at least $k$. Given that we also compute the length of a longest (not necessarily contiguous) subsequence of $S$ ending with each $S[j]$ and with every run of length at least $k$, we can select in $O(n)$ time the ending element of the subsequence we need to trace back.

In conclusion, once the $O\left(n k^{2}\right)$ time algorithm for computing the length of a longest $k$-rollercoaster is executed, we can actually compute the respective $k$-rollercoaster in $O(n)$ additional time.

For the $O\left(n \log ^{2} n\right)$ Algorithm In order to retrieve the elements of a longest $k$ rollercoaster we need to extend our algorithm to maintain global arrays Pred $_{\text {inc }}[1, \ldots, n]$ and $\operatorname{Pred}_{\mathrm{dec}}[1, \ldots, n]$. Elements of these arrays are computed during the calculations of inc $m[m: j]$ and $\operatorname{dec}_{m}[m: j]$ as follows. Initially they are equal to -1 . After execution of the algorithm we demand that Pred $\mathrm{d}_{\text {inc }}$ satisfies the following: for every $1 \leq i \leq n$ we have that inc $[i]=\operatorname{dec}\left[\operatorname{Pred}_{\text {inc }}[i]\right]+M^{\prime}\left[\operatorname{Pred}_{\text {inc }}[i]-1, i\right]-1$ if Pred $_{\text {inc }}[i] \neq-1$ and inc $[i]=\max \left\{0, M^{\prime}[0, i]\right\}$ otherwise, and similarly for Pred $\mathrm{dec}_{\text {. }}$ It is straightforward to augment the algorithm from Corollary 1 to obtain such information.

We retrieve the elements of a longest $k$-rollercoaster from the last one to the first one. Recall that a longest $k$-rollercoaster has the length equal to $\max \{\operatorname{inc}[n], \operatorname{dec}[n]\}$. We focus on how to obtain a longest $k$-rollercoaster $R$ of length inc $[n]$ with last run increasing (so, assume, w.l.o.g., that inc $[n]>\operatorname{dec}[n]$ ); the procedure is similar for $\operatorname{dec}[n]$ and the last run decreasing.

Observe that if inc $[n]$ is equal to the length of LIS in the input sequence, we can obtain the elements of $R$ by Algorithm 1 in $O(n \log n)$ time. Otherwise, there exists $i<n$ such that inc $[n]=\operatorname{dec}[i]+M[i-1, n]-1$. The value $i$ is stored in $\operatorname{Pred}_{\text {inc }}[n]$. In this case, we construct $R$ by finding recursively a longest $k$-rollercoaster associated with dec $[i]$ and concatenating it with LIS in $S[i: n]$. This holds because, by Lemma 7 the last run of $R$ is a LIS in $S[i: n]$. Obtaining LIS in $S[i: n]$ can be done in $O((n-i) \log n)$ time.

Thus, in general, we will need to compute a series of LISs and LDSs on the ranges $S\left[n_{i-1}: n_{i}\right]$, for $1 \leq i \leq m$, where $m$ is the number of runs in a longest $k$-rollercoaster, $n_{m}=n$ and $n_{0}=1$. Moreover, $n_{i-1}=\operatorname{Pred}_{\text {inc }}\left[n_{i}\right]$, if the $i^{\text {th }}$ run of the rollercoaster is increasing and $n_{i-1}=\operatorname{Pred}_{\mathrm{dec}}\left[n_{i}\right]$, if the $i^{\text {th }}$ run of the rollercoaster is decreasing. Obtaining the LIS in $S\left[n_{i-1}: n_{i}\right]$ can be done in $O\left(\left(n_{i}-n_{i-1}+1\right) \log n\right)$ time.

Adding up the time needed to compute LIS or LDS for each of these ranges we get $O(n \log n)$ total time needed to obtain elements of a longest $k$-rollercoaster.

\section{Lower Bound}

In the final section of our paper, we prove that any comparison-based algorithm computing the length of a longest $k$-rollercoaster in a permutation $S$ of $\{1, \ldots, n\}$, for 
$4 \leq k \leq \frac{n}{3}$, performs at least $\Omega(n \log k)$ comparisons. Let $T$ be a binary comparison tree associated with an algorithm that computes the result. The number of comparisons made in the algorithm in the worst case is equal to the height of $T$, and this is a lower bound on the execution time of the algorithm.

Let $A$ be a partial ordering associated with a path from the root to some leaf of $T$. Since the algorithm cannot distinguish between permutations following the same path, every permutation consistent with $A$ has to give the same result. Our approach is to first identify a set $U$ of permutations of $\{1, \ldots, n\}$ such that $\log |U|=\Theta(n \log k)$, and any ordering associated with a leaf of $T$ can be consistent with at most one permutation from $U$. Hence, the number of leaves in $T$ is at least $|U|$. Since the height of a binary tree is at least logarithm of the number of leaves, this will show that the height of $T$, and hence also the number of comparison performed by the algorithm, is at least $\Omega(\log |U|)=\Omega(n \log k)$.

We first recall the set $\Gamma$ of $\ell^{n-2 \ell}$ permutations of $\{1, \ldots, n\}$ proposed by Fredman [12], where $\ell$ is a parameter. These permutations are essentially different inputs $S$ for an algorithm computing the length of LIS, each leading to a different leaf in the comparison tree.

The idea behind the definition of $\Gamma$ is to construct input sequences $\left(x_{1}, \ldots, x_{n}\right)$, with their elements $x_{1}, \ldots, x_{n}$ chosen so that certain linear orderings of the $x_{i}$ s are induced. To create a permutation from $\Gamma$ we partition $\left(x_{1}, \ldots, x_{n}\right)$ into $\ell$ subsequences $P_{1}, P_{2}, \ldots, P_{\ell}$. To simplify the exposure, let the $\ell_{\text {prefix }}$ of a sequence be its prefix of length $\ell$, while the $\ell_{\text {suffix }}$ is its suffix of length $\ell$; the remaining $n-2 \ell$ elements are called $\ell_{\text {middle }}$ of the sequence. We partition $\left(x_{1}, \ldots, x_{n}\right)$ in the following way: the $i^{\text {th }}$ element of $\ell_{\text {prefix }}$ (that is, $\left.x_{i}\right)$ and the $i^{\text {th }}$ element of $\ell_{\text {suffix }}\left(x_{n-\ell+i}\right)$ belong to $P_{i}$. Each element from $\ell_{\text {middle }}$ of the sequence belongs to an arbitrary chosen part $P_{j}$. This gives us $\ell^{n-2 \ell}$ different partitions. For a partition $P_{1}, \ldots, P_{\ell}$, we assign values from $\{1, \ldots, n\}$ to the input sequence in such a way, that the elements of each part $P_{i}$ form a decreasing sequence and, for $1 \leq i \leq \ell-1$, each element of $P_{i}$ is less than any element of $P_{i+1}$ (see Fig. 5). So, each such possible assignment gives us a permutation from $\Gamma$. LIS of any permutation from $\Gamma$ is of length $\ell$ because it contains one element from each $P_{i}$. LDS of any permutation of $\Gamma$ is no longer than $n-2 \ell+2$ because it contains at most one element from $\ell_{\text {prefix }}$ and at most one from $\ell_{\text {suffix }}$.

Proposition 5 Each permutation from $\Gamma$ can be split into $\ell$ descending subsequences in only one way. For two different permutations from $\Gamma$ these ways of splitting are different.

Proof Let $P$ be a permutation from $\Gamma$ and $P_{1}, \ldots, P_{\ell}$ be its corresponding partition as described above. Observe that elements of $\ell_{\text {prefix }}$ (respectively, $\ell_{\text {suffix }}$ ) of $P$ form an increasing subsequence, so no two of them can be in the same decreasing subsequence. Now let $D_{1}, \ldots, D_{\ell}$ be a partition of $P$ into $\ell$ decreasing subsequences, such that $D_{i}$ contains the $i^{\text {th }}$ element from $\ell_{\text {prefix }}$. Since elements of $\ell_{\text {suffix }}$ form an increasing subsequence, each $D_{i}$ has to contain exactly one of them. Because only the first element in $\ell_{\text {suffix }}$ is smaller than the first element in the $\ell_{\text {prefix }}, D_{1}$ actually has to contain the first element of $\ell_{\text {suffix }}$. Repeating this reasoning, we obtain that $D_{i}$ contains the $i^{\text {th }}$ element from $\ell_{\text {prefix }}$ and also the $i^{\text {th }}$ element from $\ell_{\text {suffix }}$. Then, we obtain that $D_{1}$ is 


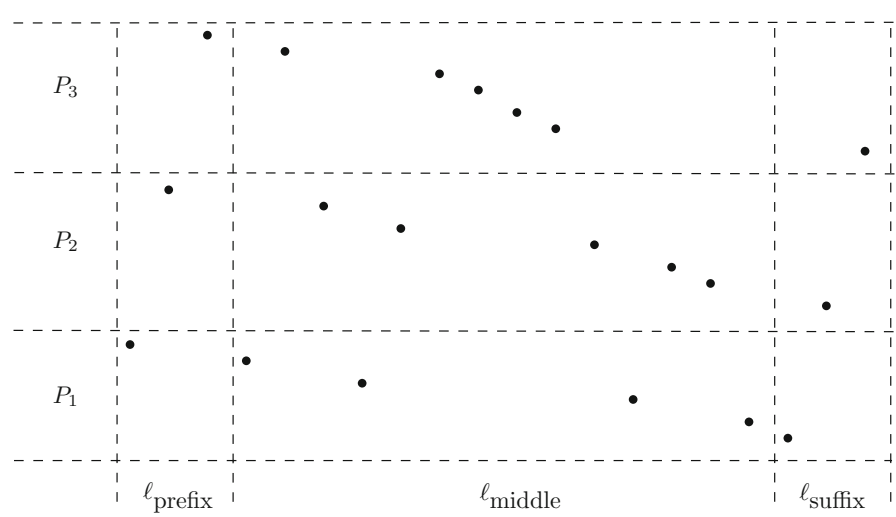

Fig. 5 Example permutation $P \in \Gamma$ for $\ell=3$ in a plane. In this figure, we have $P=$ $(6,13,20,5,19,12,4,11,18,17,16,15,10,3,9,8,2,1,7,14)$

actually equal to $P_{1}$, and by repeating this reasoning, that $D_{i}=P_{i}$ for all $i=1, \ldots, \ell$.

We now consider the algorithm computing the length of a longest $k$-rollercoaster. Using the permutations from $\Gamma$ we create a set $U$ of $k^{n \frac{k-3}{3 k-3}}$ permutations of $\{1, \ldots, n\}$, again with the same principle behind: they should be input sequences which lead to different paths in the comparison tree associated to an algorithm computing the length of a longest $k$-rollercoaster. Observe that $\log \left(k^{n \frac{k-3}{3 k-3}}\right)=\Theta(n \log k)$, so this would imply the desired lower bound of $\Theta(n \log k)$ on the number of comparisons done by an algorithm to compute the length of a longest $k$-rollercoaster.

A permutation from $U$ is obtained as follows. Suppose that $(3 k-3)$ divides $n$. Split the sequence $\left(x_{1}, \ldots, x_{n}\right)$ into $\frac{n}{3 k-3}$ blocks (contiguous subsequences) of size $3 k-3$. We will assign to the elements of the $i^{\text {th }}$ contiguous block $\left(x_{i(3 k-3)+1}, \ldots, x_{(i+1)(3 k-3)}\right)$ distinct values from the set $\{i(3 k-3)+1, \ldots,(i+1)$ $(3 k-3)\}$, as follows. In every block, use one of the permutations from $\Gamma$ (with the parameter $\ell$ set to $k$ ) to values to the elements $x_{i(3 k-3)+1}, \ldots, x_{(i+1)(3 k-3)}$ of that block, and then assign values to those elements according to that ordering. In this way, we can create $|\Gamma|^{\frac{n}{3 k-3}}=\left(k^{k-3}\right)^{\frac{n}{3 k-3}}$ permutations of $\{1, \ldots, n\}$. Observe that in every block the length of a longest decreasing subsequence is less than $k$. Since every block consists of strictly greater values than the previous ones, a longest decreasing subsequence of every permutation from $U$ is less than $k$. A longest increasing subsequence of every element of $\Gamma$ is equal to $k$, so a longest $k$-rollercoaster for every element of $U$ is equal to $\frac{k n}{3 k-3}$ and consists only of longest increasing subsequences corresponding to all the blocks glued one after the other. We can now show a result similar to Proposition 5.

Proposition 6 Each permutation from $U$ can be split into $\frac{k n}{3 k-3}$ descending subsequence in only one way. For two different permutations from $U$ these ways of splitting are different. 
Proof Let $S$ be a permutation from $U$. Recall that we can partition $S$ into $\frac{n}{3 k-3}$ contiguous blocks of length $3 k-3$. All values in a block are strictly greater than the values in all previous blocks, so in a decreasing subsequence of $S$ we can have only elements from one block. Since every block corresponds to a permutation from $\Gamma$, by Proposition 5 it can be split into exactly $k$ decreasing subsequences in only one way. For each two different permutations of $U$, there exists at least one block (i.e., permutation from $\Gamma$ ) that differentiates them. By Proposition 5, this block is split in a different way than all the other blocks of $\Gamma$, so the conclusion follows: each particular permutation from $U$ will also be split in a different way than all other permutations of $U$.

Having constructed the set $U$, we can proceed with the lower bound. Let $A$ be a partial ordering associated with a path to some leaf of $T$ (the comparison tree associated to the algorithm computing the length of a longest $k$-rollercoaster). Since the algorithm cannot distinguish between permutations following the same path, every permutation consistent with $A$ has to give the same result. We recall the following lemma.

Lemma 9 (Lemma 3.6 in [12]) Let $\leq$ be a partial ordering defined on S. The maximum length of LIS in $S$ associated with any linear embedding of this ordering, is equal to the minimum number of decreasing subsequences relative to $\leq$ into which $S$ can be partitioned.

Now we can prove the following.

Lemma 10 Let $A$ be partial ordering associated with the path from the root to a leaf of $T$. Only one permutation from $U$ can be consistent with A.

Proof Consider $S \in U$ that is consistent with $A$, and let $D=\frac{k n}{3 k-3}$ be the length of its LIS. Now let $m$ be the minimum number of decreasing subsequences relative to the results of the comparisons made on the path $A$ into which $S$ can be partitioned. If $m<d$ then $S$ is consistent with $A$, so we can partition $S$ into the same decreasing subsequences, but $S$ cannot be divided into less than than $d$ decreasing subsequences, a contradiction. If $m>d$ then by Lemma 9 there exists a permutation $S^{\prime}$ consistent with $A$ with the length of LIS greater than $d . S^{\prime}$ follows the same path as $S$ in the comparison tree, but has a longer $k$-rollercoaster (consisting only of LIS of $S^{\prime}$ ) than $S$, a contradiction. Thus, $m=d$ for any such $S$.

Consider two $S_{1}, S_{2} \in U$ consistent with $A$. By Proposition 6, the only partition of $S_{1}$ into $d$ decreasing sequences is different from the only such partition of $S_{2}$ (into $d$ decreasing sequences), so $A$ can be consistent with only one permutation, a contradiction.

Thus, each permutation from $U$ corresponds to a distinct leaf of $T$, making the depth of $T$ at least $\log |U|=\Theta(n \log k)$ as required and proving the following theorem.

Theorem 5 For every $k$ satisfying $4 \leq k \leq \frac{n}{3}$, any comparison-based algorithm that computes the length of a longest $k$-rollercoaster in a permutation of $\{1, \ldots, n\}$ performs at least $\Omega(n \log k)$ comparisons. 


\section{Conclusions}

In this paper, we presented a comparison-based algorithm computing the length of a longest $k$-rollercoaster in a sequence of $n$ distinct numbers in $O\left(n k^{2}\right)$ time. This solves in optimal linear-time the problem of computing a longest $k$-rollercoaster in an array for constant values of $k$. In particular, the problem of computing a longest rollercoaster in an array (i.e., the case $k=3$ ) is solved optimally, which solves an open problem from [6]. We also present a subquadratic algorithm that computes a longest $k$-rollercoaster in a sequence of $n$ distinct numbers in $O\left(n \log ^{2} n\right)$ time, i.e., outperforms the algorithm above, as well as other algorithms from the literature [57], for values $k=\omega(\log n)$. In the last section of the paper, we showed that any comparison-based algorithm computing a longest $k$-rollercoaster needs to perform $\Omega(n \log k)$ comparisons. We leave as an open problem to close the gap between the lower and upper bounds shown here.

Funding Open Access funding enabled and organized by Projekt DEAL. The work of Florin Manea was funded by the German Research Foundation (DFG), project MA 5725/2-1.

Open Access This article is licensed under a Creative Commons Attribution 4.0 International License, which permits use, sharing, adaptation, distribution and reproduction in any medium or format, as long as you give appropriate credit to the original author(s) and the source, provide a link to the Creative Commons licence, and indicate if changes were made. The images or other third party material in this article are included in the article's Creative Commons licence, unless indicated otherwise in a credit line to the material. If material is not included in the article's Creative Commons licence and your intended use is not permitted by statutory regulation or exceeds the permitted use, you will need to obtain permission directly from the copyright holder. To view a copy of this licence, visit http://creativecommons.org/licenses/by/4.0/.

\section{References}

1. Aggarwal, A., Klawe, M.M.: Applications of generalized matrix searching to geometric algorithms. Discrete Appl. Math. 27(1-2), 3-23 (1990)

2. Aggarwal, A., Klawe, M.M., Moran, S., Shor, P.W., Wilber, R.E.: Geometric applications of a matrixsearching algorithm. Algorithmica 2, 195-208 (1987)

3. Aldous, D., Diaconis, P.: Longest increasing subsequences: from patience sorting to the Baik-DeiftJohansson theorem. Bull. Am. Math. Soc. 36, 413-432 (1999)

4. Bespamyatnikh, S., Segal, M.: Enumerating longest increasing subsequences and patience sorting. Inf. Process. Lett. 76(1), 7-11 (2000)

5. Biedl, T.C., Biniaz, A., Cummings, R., Lubiw, A., Manea, F., Nowotka, D., Shallit, J.: Rollercoasters and caterpillars. In: ICALP, volume 107 of LIPIcs, pp. 18:1-18:15. Schloss Dagstuhl - Leibniz-Zentrum fuer Informatik (2018)

6. Biedl, T.C., Biniaz, A., Cummings, R., Lubiw, A., Manea, F., Nowotka, D., Shallit, J.: Rollercoasters: Long sequences without short runs. SIAM J. Discrete Math. 33(2), 845-861 (2019). (This extends the conference paper [5])

7. Biedl, T.C., Chan, T.M., Derka, M., Jain, K., Lubiw, A.: Improved bounds for drawing trees on fixed points with L-shaped edges. In: Graph Drawing and Network Visualization-25th International Symposium, GD 2017, Revised Selected Papers, volume 10692 of Lecture Notes in Computer Science, pp. 305-317. Springer (2017)

8. Chazelle, B.: A functional approach to data structures and its use in multidimensional searching. SIAM J. Comput. 17(3), 427-462 (1988)

9. Crochemore, M., Porat, E.: Fast computation of a longest increasing subsequence and application. Inf. Comput. 208(9), 1054-1059 (2010)

10. Dilworth, R.P.: A decomposition theorem for partially ordered sets. Ann. Math. 51(1), 161-166 (1950) 
11. Erdős, P., Szekeres, G.: A combinatorial problem in geometry. Compos. Math. 2, 463-470 (1935)

12. Fredman, M.L.: On computing the length of longest increasing subsequences. Discrete Math. 11(1), 29-35 (1975)

13. Gajewska, H., Tarjan, R.E.: Deques with heap order. Inf. Process. Lett. 22(4), 197-200 (1986)

14. Hunt, J.W., Szymanski, T.G.: A fast algorithm for computing longest common subsequences. Commun. ACM 20(5), 350-353 (1977)

15. Kitaev, S.: Patterns in Permutations and Words. Springer, Berlin (2011)

16. Linton, S., Ruškuc, N., Vatter, V. (eds.): Permutation Patterns. London Mathematical Society Lecture Note Series, vol. 376. Cambridge (2010)

17. Romik, D.: The Surprising Mathematics of Longest Increasing Subsequences. Cambridge (2015)

18. Schensted, C.: Longest increasing and decreasing subsequences. Can. J. Math. 13, 179-191 (1961)

19. Steele, J.M.: Variations on the monotone subsequence theme of Erdôs and Szekeres. In: Aldous, D., Diaconis, P., Spencer, J., Steele, J.M. (eds.) Discrete Probability and Algorithms, pp. 111-131. Springer, New York (1995)

20. Sun, X., Woodruff, D.P.: The communication and streaming complexity of computing the longest common and increasing subsequences. In: Proceedings of the Eighteenth Annual ACM-SIAM Symposium on Discrete Algorithms, SODA '07, pp. 336-345. Society for Industrial and Applied Mathematics (2007)

21. Tiskin, A.: Fast distance multiplication of unit-Monge matrices. Algorithmica 71(4), 859-888 (2015)

22. Weimann, O.: Accelerating Dynamic Programming. PhD thesis, Massachusetts Institute of Technology, Cambridge, MA, USA (2009)

Publisher's Note Springer Nature remains neutral with regard to jurisdictional claims in published maps and institutional affiliations. 